\section{Recall Injection-Site Reactions After Treatment With Anakinra}

De Aramburu Mera T, Reguero Capilla M, Ochando Diez-Canseco M, Prados Castaño M

Department of Allergology, University Hospital Virgen del Rocio, Seville, Spain

J Investig Allergol Clin Immunol 2021; Vol. 31(3): 275-276 doi: $10.18176 /$ jiaci.0642

Key words: Recall injection-site reactions. Anakinra. Canakinumab. TRAPS

Palabras clave: Reacción local. Anakinra. Canakinumab. TRAPS.

Periodic tumor necrosis factor receptor-associated periodic syndrome (TRAPS) is a very rare hereditary autoinflammatory disorder caused by a mutation that is located primarily in exons 2 to 4, which encode the first or second cysteine-rich extracellular domains, resulting in unregulated systemic inflammation. Common signs and symptoms of TRAPS include recurrent fever, conjunctivitis, arthritis, chest pain, pericarditis, myalgia, and an erythematous rash. In the absence of an underlying infection, malignancy, or other autoimmune disorders, the most common clinical manifestation of TRAPS is recurrent fever.

Corticosteroids help limit acute flare-ups, although only as a temporary measure. Maintenance therapy with biological agents is used for more severe cases. Beneficial effects of anakinra and tocilizumab have been demonstrated [1]

Anakinra is an IL-1 receptor antagonist that acts by blocking the biological activity of naturally occurring IL-1, thus competitively inhibiting binding of IL-1 to its receptor. Canakinumab is a human monoclonal antibody that specifically and selectively blocks IL-1ß. Both anakinra and canakinumab are used as treatment for autoinflammatory syndromes and TRAPS [2,3].

Injection site reactions (ISRs), often defined as a constellation of symptoms including swelling, erythema, pruritus, and pain around the injection site, are a common adverse event associated with various kinds of biologic therapies $[4,5]$.

Clinical experience indicates that there are 2 types of ISRs in relation to injections of anakinra. One is immediate, with a stinging and burning feeling, and the other is delayed, presenting with rash, swelling, and pain. Up to $70 \%$ of patients who receive an anakinra injection experience an ISR, and $95 \%$ of these cases are mild to moderate in severity. Onset of ISRs is generally within the first weeks of initiating therapy, and patients who do not experience an ISR within 4 weeks are unlikely to experience any ISR. The delayed type can present as a more severe reaction involving erythema, pruritus, swelling, and pain (ie, inflammatory lesions). The delayed reaction may be treated with topical corticosteroids or antihistamines and, in the vast majority of cases, disappears within 2 months.
Recall urticaria (RU) could be considered an example of such an ISR. RU is a rare biological phenomenon characterized by hives at a previously injected site when the patient is reexposed [6]. The most common example is patients who have previously received subcutaneous allergen immunotherapy and experience a local skin reaction at the sites of previous allergen injections when the allergen is administered again at a different site or after significant environmental exposure to the allergen [7]. Other stimuli such as heparin and nonsteroidal anti-inflammatory drugs have also been reported [8].

We present the case of a 29-year-old woman diagnosed with TRAPS and receiving daily treatment with subcutaneous anakinra $100 \mathrm{mg}$, which led to good control of symptoms. The patient self-administered the treatment by varying the location of the injections every day. At approximately 12 hours after the ninth injection of anakinra, she developed a hive on the left hypochondrium, which proved to be intensely itchy. After 60 minutes, 8 additional hives appeared at the previous anakinra injection sites. The treatment was interrupted and antihistamines were given. The pruritus disappeared but not the lesions, which remained as hyperpigmented flat residual brownish macules that vanished after 2 weeks (Figure).

Taking into account the symptoms, the appearance of pruritic hives points to an immediate reaction. However, the duration of hyperpigmented lesions for 2 weeks suggests a late response. Thus, in this particular case, the reaction seemed to combine immediate and late responses. We performed the followings tests: (1) Patch tests $(5 \%, 10 \%$, and $20 \%$ in petrolatum) with anakinra, canakinumab, and polysorbate (excipient present

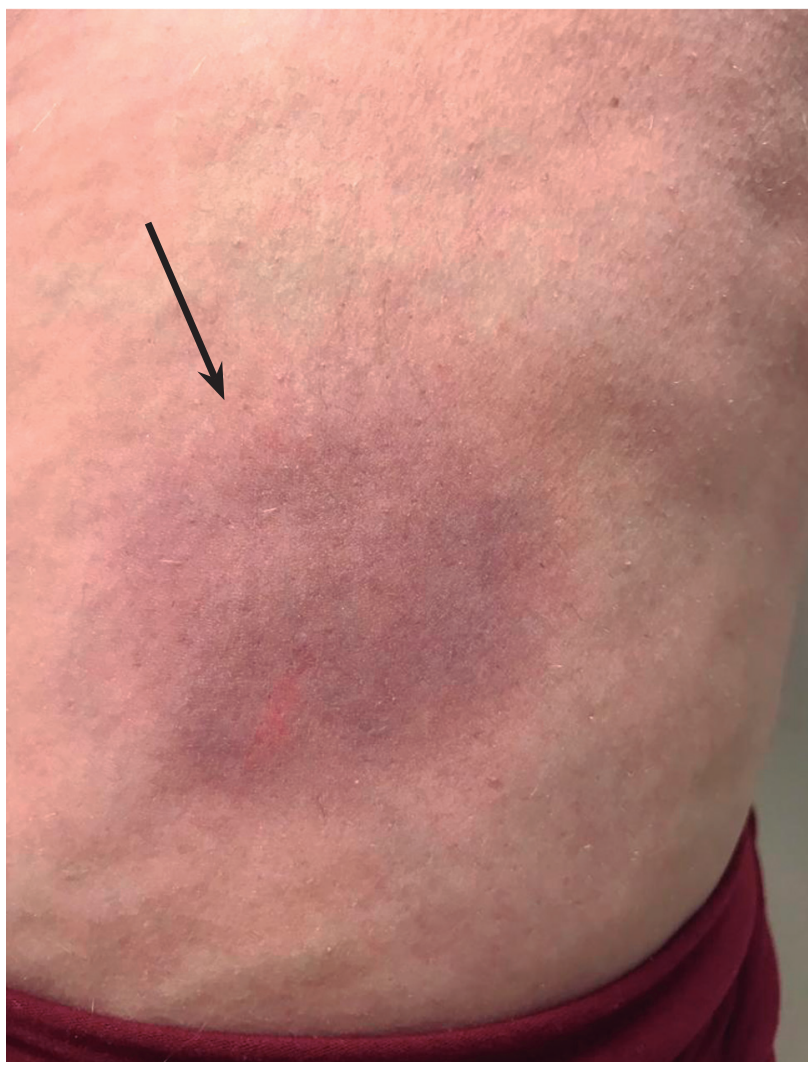

Figure. Hyperpigmented flat residual brownish macule. 
in both drugs). The patches were applied both on the injured area and on healthy skin. The readings were negative after 48 and 96 hours; (2) Skin prick and intradermal tests with the same drugs (skin prick test $0.15 \mathrm{mg} / \mathrm{mL}$; intradermal test $1 / 10$, $1 / 100$, and $1 / 1000$ ), which yielded negative results; (3) The lymphocyte activation test against anakinra, polysorbate, and canakinumab, which yielded negative results; (4) Laboratory tests (including complete blood count, comprehensive metabolic panel, coagulation tests, inmunoglobulins, total serum protein, serology including hepatotropic viruses, and cryoglobulins), which yielded normal results; (5) Biopsy of injured skin and healthy skin, which also yielded normal results; (6) Open provocation test with canakinumab (150 mg subcutaneous), in which there was no reaction. After this test, the patient received canakinumab (150 mg/4 wk). Her disease is now controlled, with no adverse reactions to the drug. The anakinra challenge test was rejected by the patient.

The case presented above is an example of an anakinrainduced ISR in a patient with TRAPS. The pathophysiological mechanism of this patient is quite controversial.

On one hand, the initial symptoms, ie, hives at the previous drug administration sites, favor the idea of anakinra-induced RU. These reactions may be mediated by IgE, T cells, or enzymatic inhibition of the cyclooxygenase type I isoform, which may play a role in the development of this specific reaction $[8,9]$. In addition, polysorbate, the component that carries both anakinra and canakinumab, has the power to induce mast cell degranulation. Another biological drug, adalimumab, has been reported to cause RU and yielded a positive result in skin testing in a patient with spondyloarthritis [10]. Unlike the present case, the underlying disease was different, the skin tests were positive, and treatment was not replaced by another biological treatment with the same function. Results for the vehicle used, polysorbate, were negative in the skin, intradermal, and patch tests. Polysorbate is also present in canakinumab, which was subsequently tolerated by the patient we report. Furthermore, the patient developed residual hyperpigmented lesions, which are not characteristic of RU.

The late phase reaction in the present case could point to fixed drug exanthema, which frequently leaves residual pigmentation and whose main characteristic is reoccurrence at the same site. Type IV hypersensitivity phenomena are involved in this condition, and intraepidermal CD8 lymphocytes play a leading role. Of particular interest is the sudden appearance of the 9 lesions after the ninth injection, days after the previous ones. However, in order to address this possibility, we performed the lymphocyte activation test, patch tests, and a biopsy study of both healthy and injured skin, all of which yielded negative results.

It seems that various mechanisms can lead to RU. Residual hyperpigmentation persisted after the disappearance of the hives. Several other mechanisms may have been involved, or patients with TRAPs may be more susceptible to this type of reaction.

We showed the patient to be tolerant to canakinumab, suggesting that the reaction is selective to anakinra and not due to blockade of IL-1 or its receptor. Therefore, to our knowledge, this is the first report of a biphasic recall ISR after treatment with anakinra in a patient with TRAPS who tolerated canakinumab, which controlled her symptoms. Further studies are needed to determine the exact pathophysiological mechanism.

\section{Funding}

The authors declare that no funding was received for the present study.

\section{Conflicts of Interest}

The authors declare that they have no conflicts of interest.

\section{Previous Presentation}

This work was presented as an e-poster at National Congress Allergology, Gran Canarias, 2019.

\section{References}

1. Verbsky JW. When to suspect autoinflammatory/recurrent fever syndromes. Pediatr Clin North Am. 2017;64:111-25.

2. Ter Haar N, Lachmann H, Özen S, Woo P, Uziel Y, Modesto C, et al. Treatment of autoinflammatory diseases: results from the Eurofever Registry and a literature review. Ann Rheum Dis. 2013; 72(5):678-85.

3. De Benedetti $F$, Gattorno $M$, Anton J, Ben-Chetrit E, Frenkel J, Hoffman HM, et al. Canakinumab for the Treatment of Autoinflammatory Recurrent Fever Syndromes. N Engl J Med. 2018;378:1908-19.

4. Kaiser C, Knight A, Nordström D, Petterson T, Fransson J, Florin-Robertsson E, et al. Injection-site reactions upon Kineret (anakinra) administration: experiences and explanations. Rheumatol Int. 2012;32:295-9.

5. Clarke JB. Mechanisms of adverse drug reactions to biologics. Handb Exp Pharmacol. 2010;(196):453-74.

6. Karaayvaz M, Ozangüç N. Recall urticaria: a case report. J Allergy Clin Immunol. 1996;97:1419-20.

7. Ta V, White AA. An unusual case of recurrent "recall urticaria" in a patient on immunotherapy. J Allergy Clin Immunol Pract. 2014;2(4):459-60.

8. Cimbollek S, Ávila-Castellano MR, Labella M, Baynova K, Aramburu T, Quiralte J. Recall Urticaria: Aspirin Also Induces It. J Investig Allergol Clin Immunol. 2018;8:131-2.

9. Picard M, Galvão VR. Current knowledge and management of hypersensitivity reactions to monoclonal antibodies. J Allergy Clin Immunol Pract. 2017;5(3):600-9.

10. Li PH, Watts TJ, Lui MS, Lau CS, Chung HY. Recall Urticaria in Adalimumab Hypersensitivity. J Allergy Clin Immunol Pract. 2018;6:1032-3.

- Manuscript received October 11, 2020; accepted for publication February 1, 2021.

Teresa De Aramburu Mera

Department of Allergology, University Hospital "Virgen del Rocío"

C/. Manuel Siurot

41013, Sevilla, Spain

E-mail: teresadearamburumera@gmail.com 